\title{
Comment on article by Milenkovic et al.: lateral tibial plateau fractures-functional outcomes and complications after open reduction and internal fixation
}

\author{
Zhi-cheng Pan ${ }^{1} \cdot$ Jing Shen ${ }^{2} \cdot$ Wei-feng Ji ${ }^{2}$
}

Received: 20 November 2020 / Accepted: 27 November 2020 / Published online: 8 January 2021

(C) SICOT aisbl 2021

Dear Editor,

We read with great interest the article "Lateral tibial plateau fractures-functional outcomes and complications after open reduction and internal fixation" by Milenkovic et al. [1]. In addition to appreciating the author's efforts, we also point out some problems, which we consider will contribute to the validity of this research.

1. There is no mention of how to deal with lateral tibial plateau fractures with ligament injuries. What we are concerned about is whether a primary ligament repair is needed. Proverbially, LCL is of great significance to the lateral stability of the knee joint, so will the manner and timing of ligament management affect functional outcomes or the occurrence of post-operative traumatic arthritis?

2. What measures should be taken to determine meniscus injury? MRI examination preoperation or under direct vision during operation? Our experience, which is similar with report, is that meniscus repair or meniscectomy should be performed during the operation after the meniscus injury or incarceration is determined with the assistance of arthroscopy [2]. In addition, the degree of platform collapse can also be determined under arthroscopy,

Wei-feng Ji

jiweifeng1230@163.com

Zhi-cheng Pan

zzypanzc@163.com

Jing Shen

appigy@126.com

1 The First Clinical Medical College, Zhejiang Chinese Medical University, Hangzhou 310006, China

2 Department of Orthopaedics, The First Affiliated Hospital of Zhejiang Chinese Medical University, 54 Youdian Road, Shangcheng District, Hangzhou 310006, Zhejiang, China so as to restore the flatness of the articular surface more accurately during ORIF.

3. We are concerned about that the study reported a higher infection rate, DVT incidence, and no compartment syndrome $[1,3]$. Whether these are related to the small sample size in ten years and other factors, the author did not analyze. In addition, the study method did not describe whether 41 patients were treated by the same team, and whether their post-operative complications and eventual functional recovery improved with the accumulation of experience of the team. As the literature says: one of the key factors for achieving anatomical reduction of the fracture and stable internal fixation is surgical experience.

4. This is our experience: after the injury, the "ORIF" should be given to the emergency department as soon as the soft tissue and skin conditions permit. The interval from injury to surgery, generally, is less than six hours. Ligament repair is usually performed in stage I after "ORIF." The incidence of infection, wound dehiscence, and DVT after surgery was $10 \%$, $3.4 \%$, and $6.7 \%$. The incidence of traumatic arthritis and final functional outcomes has not been followed up temporarily.

\section{References}

1. Milenkovic S, Mitkovic M, Mitkovic M, Stojiljkovic P, Stojanovic M (2020) Lateral tibial plateau fractures-functional outcomes and complications after open reduction and internal fixation. Int Orthop. https://doi.org/10.1007/s00264-020-04763-y

2. Abdel-Hamid MZCC, Chan YS, Lo YP et al (2006) Arthroscopic evaluation of soft tissue injuries in tibial plateau fractures: retrospective analysis of 98 cases. Arthroscopy 22:669-675

3. Manidakis N, Dosani A, Dimitriou R, Stengel D, Matthews S, Giannoudis P (2010) Tibial plateau fractures: functional outcome and incidence of osteoarthritis in 125 cases. Int Orthop 34(4):565-570

Publisher's note Springer Nature remains neutral with regard to jurisdictional claims in published maps and institutional affiliations. 